\title{
Report of the American Society of Transplantation Conference on Immunosuppressive Drugs and the Use of Generic Immunosuppressants
}

\author{
Rita R. Alloway ${ }^{a}$, Ross Isaacs ${ }^{b}$, Kathleen Lake ${ }^{c}$, \\ Peter Hoyerd , Roy First ${ }^{e}$, Harold Helderman ${ }^{f}$, \\ Suphamai Bunnapradist ${ }^{\mathrm{g}, \mathrm{j}}$, Alan Leichtman ${ }^{\mathrm{c}}$, \\ M. William Bennett ${ }^{\text {h }}$, Amir Tejani ${ }^{i}$ \\ and Steven K. Takemoto ${ }^{\mathrm{j}, *}$ \\ a University of Cincinnati, College of Medicine, Cincinnati \\ $\mathrm{OH}$ \\ ${ }^{\mathrm{b}}$ Center for Improvement of Minority Health, University of \\ Virginia Health System, Charlottesville, VA \\ c University of Michigan, Ann Arbor, MI \\ dPediatric Nephrology, University of Essen, Essen, \\ Germany \\ e Fujisawa Healthcare Inc, Deerfield, IL \\ ${ }^{\mathrm{f}}$ Vanderbilt Transplant Center, Nashville, TN \\ ' Cedars-Sinai Medical Center, Los Angeles, CA \\ h Legacy Good Samaritan Hospital, Portland, OR \\ 'New York Medical College, Valhalla, NY \\ 'David Geffen School of Medicine, University of California \\ Los Angeles, Los Angeles, CA \\ *Corresponding author: Steven K. Takemoto, \\ stakemoto@mednet.ucla.edu
}

Considerable economic and health-related costs are associated with the life-long maintenance immunosuppressive therapy required to prevent transplant rejection. Generic medications have the potential of providing equivalent therapeutic efficacy at a lower economic cost. In 2001, the American Society of Transplantation invited experts to review the data and issues associated with the approval and use of generic immunosuppressants. A summary of that meeting is reported here.

The generic medication approval process has been in effect for more than $\mathbf{3 0}$ years. All marketed generic cyclosporin formulations have met FDA criteria demonstrating bioequivalence in healthy subjects, and some were also tested in transplant recipients.

Most participants agreed that generic narrow therapeutic index immunosuppressive agents provide adequate de novo immunosuppression in low-risk transplant recipients. However, some participants expressed concern regarding the currently unquantified risk that may be associated with switching immunosuppressive agents under uncontrolled circumstances. There was broad agreement among the participants that generic medications should be clearly labeled and distinguishable from innovator drugs, and that patients should be educated to inform their physicians of any switch to or among generic alternatives. There was also strong support in favor of requiring studies to demonstrate bioequivalence in potentially at-risk patient populations, specifically African-Americans and pediatric patients.

Key words: Bioequivalence, cyclosporin, generic, transplantation

Received 15 April 2003, revised 6 May 2003 and accepted for publication 18 May 2003

\section{Introduction}

Although, transplantation is the treatment of choice for the majority of patients with end-stage renal, heart and liver disease, medication side-effects and the cost of life-long immunosuppression diminishes the quality of life for successful long-term solid organ transplant recipients.

Generic medications offer the advantage of providing equivalent therapeutic efficacy at a lower cost to the patient, healthcare system and society. Although the approval process has been in place for more than 30 years, generic substitution remains a topic of heated debate among health care professionals, members of the pharmaceutical industry, consumers, and government officials. These debates are often fueled by a lack of understanding of the mechanics of the generic drug approval process and the scientific rigor underpinning the methodology (1). At the core of this controversy is whether the current FDA standards regulating bioequivalence are restrictive enough to ensure that generic formulations of critical dose drugs are clinically equivalent to their brand name counterparts. The FDA defends these standards vigorously while the manufacturers of brand name drugs have attempted to discredit the methodology.

In the 2001, the American Society of Transplantation invited experts to address in a scientific forum the data and issues associated with approval and use of generic immunosuppressant equivalents. Preparation of this manuscript was delayed because of the untimely death of a key organizer of this meeting, Dr Tejani. This report describes 


\section{Alloway et al.}

concerns raised by health care providers in transplantation and explains important issues involved in the use and substitution of immunosuppressive agents with generic alternatives.

\section{Clinical Implications in Transplantation}

Generic products for azathioprine and prednisone have been available for a number of years. Although Imuran's patent expired in 1979, a generic formulation was not FDA approved until 1996. The introduction of generic azathioprine occurred with little fanfare, as most kidney transplant centers had previously adopted mycophenolate mofetil as the preferred antiproliferative agent in triple therapy immunosuppressive regimens. Two retrospective studies in the literature evaluating outcomes with generic azathioprine and identified no differences between formulations $(2,3)$. The patent for the chemical entity of cyclosporin expired in 1995 paving the way for the development of alternative cyclosporin formulations. Controversy arose over concerns of therapeutic failure, a lack of studies addressing safety and efficacy, absence of long-term follow-up data for generic alternatives, the recognized existence of intra- and interpatient variability in the absorption and metabolism of cyclosporin products, the lack of physician control over products dispensed to patients when generic formulations are available, and pressure associated with maintaining brand name loyalty.

\section{Generic Product Approval Process}

The generic drug approval process has evolved over the past 30 years (4). In 1970, the FDA established the Abbreviated New Drug Application (ANDA) as a mechanism for the review and approval of generic versions of drug products that had been approved between 1938 and 1962 $(1,5)$. For drugs approved after 1962, manufacturers of generic products were required to submit complete safety and efficacy data. After 1978 manufacturers were required to cite published reports of trials documenting safety and efficacy. Neither of these approaches was acceptable because of the expense incurred in undertaking clinical studies and the lack in many cases of published data. In 1984, the Drug Price Competition and Patent Term Restoration Act focused on modifying and accelerating the ANDA procedure and gave the FDA statutory authority to approve generic versions of innovator products approved after 1962 as safe and effective $(1,5-7)$. The revised ANDA process does not require manufacturers to include preclinical or clinical data establishing the safety and efficacy of the active ingredients $(1,5)$ because these data were previously documented during the approval process for the innovator product.

A generic drug product can be substituted for an innovator drug product based on the belief that the two products are pharmaceutically equivalent as well as bioequivalent. To be considered pharmaceutically equivalent, two drug products must contain identical amounts of the same active ingredient in the same dosage form, be formulated to meet the same compendia standards of quality and purity, and generally be labeled for the same indications. However, pharmaceutical equivalents may differ in terms of the excipients they contain, their shape, scoring, packaging, and in certain circumstances labeling.

Bioequivalence refers to the absence of a significant difference in the rate and extent to which the active ingredients in pharmaceutical equivalents become available at the site of drug action in the body when administered under similar experimental conditions (1,8-10). The surrogate clinical endpoints for bioequivalence studies are based upon pharmacokinetic parameters of the active ingredient for rate and extent of absorption, Tmax, Cmax, and AUC. This assumption implies that if the active ingredient of the generic product is absorbed and becomes available at the site of action at equivalent concentrations as the innovator, then the clinical effect is the same. This same assumption also exists for different formulations of a drug (e.g. once cyclosporin reaches its site of action, it has the same pharmacologic activity regardless of whether it was delivered by the Sandimmune, Neoral, or a generic equivalent formulations or whether it was prescribed as a capsule or the solution).

Assessment of bioequivalence is central to the ANDA process and is what differentiates the ANDA from a NDA. By design, bioequivalence studies do not have a clinical endpoint. Similar plasma concentration time-profiles are taken as a surrogate for therapeutic efficacy and safety $(11,12)$. The concept of bioequivalence is based on the relationship expected between the time course and concentration achieved in blood after the administration of a drug and its expected clinical effect. To be considered as bioequivalent under the Federal Food, Drug, and Cosmetic Act, the rate and extent of absorption of the generic drug cannot be significantly different from the rate and extent of absorption of the brand name drug when administered at the same dose under similar circumstances (13).

A bioequivalence study is typically conducted with 1836 healthy human subjects. They should be older than 18 years of age and represent a reasonable representation of different ages, ethnicities, and gender $(1,8,14,15)$. Performing initial bioequivalence studies in healthy volunteers is a safe stepwise approach to generic drug testing, and does not preclude further testing in the target population. Although some have argued that it may be more appropriate to assess bioequivalence in patients who are likely to receive the drug, this strategy has potential limitations of exposing patients to bioinequivalent products. Children younger than 18 years of age are excluded from these studies because of concerns about obtaining meaningful informed consent from minors and the medical ethics of 
administering pharmacologic agents to healthy children. The timing and number of plasma samples collected must be adequate to define at least $80 \%$ of the total area under the time-concentration curve. It is recommended that the sampling continue for a period of at least three times the terminal half-life of the active ingredient $(1,8,10,15)$. From these constructed time-concentrations curves, bioequivalence is calculated using three pharmacokinetic parameters: the maximum concentration (Cmax), the time at which the maximum concentration is reached (Tmax), and the area under the time vs. concentration curve (AUC). The rate of absorption is evaluated by measuring the $\mathrm{Cmax}$ and the Tmax. The extent of absorption is calculated by measuring the AUC. Manufacturers may choose to conduct a pilot bioequivalence study to estimate this variability, or they may rely on values reported in the literature to estimate sample size (1). The appropriate sample size is based upon the assessment of average bioequivalence and determined using published formulas of alpha $=0.05$ and power $=90 \%$.

Other questions arise from the lack of individual bioequivalency data, especially as it relates to certain subsets of a population. Draft guidance has been proposed by the FDA to measure individual bioequivalence, but these criteria have not been statistically validated. This study design proposes replicate administration in four period crossover studies of both the innovator and the test product, thus allowing the calculation of both the inter- and intrapatient variability of the two products tested.

It is important to recognize that bioequivalence studies are conducted for a variety of reasons, which include demonstrating equivalence between agents when there have been manufacturing and/or formulation changes by the innovator manufacturer of the original product. Often the product the innovator company used during clinical trials differs from the final marketed medication. In fact, of the 97 oral agents approved by the FDA as new molecular entities from 1981 to 1990 only 38\% of the marketed formulations were precisely the same as those tested in the pivotal Phase III trials. The remaining medications were marketed in a different formulation from that studied in the clinical trials. These formulations were licensed based upon bioequivalency studies comparing the final marketed product to that administered during the clinical trials of the active ingredient (16).

Additional reasons exist for formulation and dosage form changes other than development of generic alternatives. When minor manufacturing or dosage form changes are made, it is only necessary for the new product to demonstrate bioequivalence to the original formulation described in the NDA. Specific examples of this in transplantation include the introduction by the innovator manufacturer of Sandimmune capsules, and various new formulations and alternative strengths of mycophenolate mofetil, tacrolimus and Neoral. These products, like generic alternatives, con- tained the same active ingredient, but differed in various excipients and other ingredients (11,17-21).

A number of medications requiring therapeutic drug monitoring have met the FDA's bioequivalency standards and are available in generic formulations. In the case where a generic product exists, selection of the product dispensed may be controlled by the patient, the patient's pharmacy benefits organization (or third party insurer), the physician and/or by the pharmacist. Currently, all US states and territories have some form of drug product selection law allowing pharmacists, under certain conditions, to substitute a generic product when a physician has written a prescription using the brand name (11). A table summarizing the variations in applicability of the state drug product selection laws has been published (11). In addition there are published recommendations for the use of alternative cyclosporin formulations (11).

\section{Unique Issues in Transplantation}

There are considerable costs associated with the life-long maintenance immunosuppressive therapy required to prevent transplant rejection $(11,22,23)$. In addition one needs to consider the costs of the treatment for potential complications or therapeutic failures. The cost of drugs during the first year following kidney transplantation is responsible for approximately $15-25 \%$ of transplant-related expenditures billed to Medicare, increasing to $30-90 \%$ for subsequent years following transplantation (24). In the United States, the patent for cyclosporin expired in September 1995. This opened the doors to the development of several alternative cyclosporin formulations. However, patent protection remains in effect over the specific formulation of Neoral.

All applications for an ANDA for cyclosporin modified must demonstrate at a minimum bioequivalence in healthy volunteers under fasted states. When scientific studies or the innovator product label identifies an effect of food on absorption or administration, bioequivalency studies must be performed in the fed state as well. In these trials, the pharmacokinetic parameters of reference and the innovator product are compared under the same fed conditions. To date, the following ANDA applications for cyclosporin-modified products have been approved; SangCya liquid (SangStat Medical Corporation, Fremont CA, USA), cyclosporin soft gelatin capsules, modified (Eon Labs, Inc., Laurelton, NY, USA), Gengraf capsules and liquid (SangStat), and cyclosporin soft gelatin capsules and liquid, modified (PLIVA, Inc., East Hanover, NJ, USA). All generic formulations of cyclosporin-modified capsules have met FDA criteria for approval as AB-rated generics. However, SangCya oral solution was withdrawn from the market because it was not bioequivalent in apple juice. Two generic liquid cyclosporin-modified products are currently commercially available. Each product has undergone extensive testing with orange and apple juice, 


\section{Alloway et al.}

however, just as with Neoral, patients should exercise caution and consistency when mixing cyclosporin-modified liquid with nontested vehicles.

Two studies addressed the issues of safety and efficacy of switching formulations after initial dosage titration. In a four period replicate designed trial comparing SangCya and Neoral in orange juice, no interindividual or intraindividual variances were noted (11). A trial comparing Gengraf to Neoral pharmacokinetic profiles at steady state and immediately post conversion in stable renal transplant patients also did not find any significant differences $(25,26)$.

\section{Summary of Conference Proceedings}

A central question of the conference focused on whether standard bioequivalence criteria used by the FDA are sufficiently robust to address the issues that are unique to transplantation (11,27-29). Several factors influence cyclosporin pharmaco kinetics in transplant patients, including age, disease state, race, ethnicity, gender, diet, and intra- and interindividual variation in metabolism and enteric transport processes. Absorption and metabolism of cyclosporin, tacrolimus, sirolimus, corticosteroids and other medications used in transplantation dependent upon gut transport and metabolism (30). Polymorphisms of these enzymes help explain intra- and interpatient variability in blood levels. Many foods and medications inhibit or induce the ability of these enzymes and transport proteins to perform their absorptive or metabolic functions $(31,32)$.

Non-compliance is a prominent cause of allograft failure. At least part of this noncompliance has been attributed to the inability of patients to afford expensive immunosuppressive medications (33). If savings resulting from the use of generic immunosuppressive medications are passed on to payers and consumers, then the use of generic alternatives could potentially result in a reduction in out-of-pocket costs and perhaps in improved compliance.

Most observers agreed that the de novo prescription of immunosuppressive generic medications is safe as long as the generic is bioequivalent as defined by the FDA and, as with the use of the innovator products, appropriate therapeutic drug monitoring is undertaken when indicated.

A uniform opinion could not be reached regarding the safety of switching among cyclosporin formulations without incremental monitoring. There was a support for recommendations that physicians and patients should be notified of any switch in dispensed brands, that there should be pill and container uniqueness among generic alternatives, that patients should be educated to inform their physicians when a switch has taken place so that tests to assure that adequate blood levels are achieved could, when indicated, be performed, and that different formulations should not be mixed together until data supporting the safety and efficacy of such a practice is developed.
Theoretical concerns still exist regarding the use of bioequivalent cyclosporin-modified products in the pediatric population. Pharmacokinetic studies of cyclosporin in children have demonstrated a faster absorption rate, higher peak concentrations, and more rapid clearances when compared with adults (34-37), but these pharmacokinetic differences have not been shown to be product dependent. To date, there have been no formal studies evaluating bioequivalence of the cyclosporin-modified generic products in children.

Ethnic differences exist in the effectiveness, absorption, metabolism and recommended dosages of critically dosed therapeutic immunosuppressive agents $(29,38)$. Current recommendations on the use of generic cyclosporin in US minority transplant recipients are based on small numbers of patients with limited short- or long-term outcome data $(25,26)$. The underserved and minority renal failure populations in the US are theoretically at the greatest risk for nonadherence resulting from the costs of immunosuppressive medications. Assuming that savings are passed on to the patients and society, these underserved populations potentially have the most to gain from the introduction of generic immunosuppressive medications.

\section{Consensus Points}

1 Welfare of the individual patient should be the preeminent concern in any prescribing or dispensing decision.

2 Participants strongly support the availability of efficacious, less expensive, immunosuppressive medications and endorse efforts to introduce generic alternatives. Medication costs may contribute to noncompliance with prescribed medical regimens.

3 With appropriate therapeutic drug monitoring, FDAapproved generic narrow therapeutic index immunosuppressive agents appear to provide adequate immunosuppression to low-risk transplant recipients.

4 Currently there are insufficient data to make separate recommendations regarding the use of generic immunosuppressive medications in potentially at-risk patient populations, specifically African-Americans or pediatric patients.

Demonstrations of bioequivalence in at-risk populations should be incorporated into the generic drug approval process. Consistency of the immunosuppressant brand, formulation, timing of drug administration, and blood level monitoring, along with the many other variables potentially effecting the resulting target drug concentration, are encouraged.

\section{References}

1. Welage LS, Kirking DM, Ascione FJ, Gaither CA. Understanding the scientific issues embedded in the generic drug approval process. J Am Pharm Assoc (Wash) 2001; 41: 856.

American Journal of Transplantation 2003; 3: 1211-1215 
2. Cuffari C, Hunt S, Bayless TM. Enhanced bioavailability of azathioprine compared to 6-mercaptopurine therapy in inflammatory bowel disease: correlation with treatment efficacy. Aliment Pharmacol Ther 2000; 14: 1009.

3. Haroldson JA, Somerville KT, Carlson S, Hanson J, Emery RW, Lake KD. A retrospective assessment of safety, efficacy, and pharmacoeconomics of generic azathioprine in heart-transplant recipients. J Heart Lung Transplant 2001; 20: 372.

4. Nightingale SL, Morrison JC. Generic drugs and the prescribing physician. JAMA 1987; 258: 1200

5. Henderson J, Dighe S, Williams R. Subject selection and management in bioequivalence studies. Clin Res Reg Aff 1992; 9: 71.

6. Gleiter $\mathrm{CH}$, Gundert-Remy U. Bioinequivalence and drug toxicity. How great is the problem and what can be done? Drug Saf 1994; 11: 1 .

7. Rheinstein $\mathrm{PH}$. The generic drug approval process. Am Fam Physician 1993; 48: 1357.

8. Nation RL, Sansom LN. Bioequivalence requirements for generic products. Pharmacol Ther 1994; 62: 41.

9. Skelly JP. Bioavailability and bioequivalence. J Clin Pharmacol 1976; 16: 539

10. Patnaik R, Lesko LJ, Chan K, Williams RL. Bioequivalence assessment of generic drugs. an American point of view. Eur J Drug Metab Pharmacokinet 1996; 21: 159.

11. Christians U, First MR, Benet LZ. Recommendations for bioequivalence testing of cyclosporine generics revisited. Ther Drug Monit 2000; 22: 330.

12. Garbe E, Rohmel J, Gundert-Remy U. Clinical and statistical issues in therapeutic equivalence trials. Eur J Clin Pharmacol 1993; 45: 1 .

13. Federal Food, Drug, Cosmetic Act 21 USC, 355 (j)\&(B).

14. Marzo A, Balant LP. Bioequivalence. An updated reappraisal addressed to applications of interchangeable multi-source pharmaceutical products. Arzneimittelforschung 1995; 45: 109

15. Bioavailability and Bioequivalence Requirements. 21 CFR 320.163, 2001.

16. Benet LZ, Goyan JE. Bioequivalence and narrow therapeutic index drugs. Pharmacotherapy 1995; 15: 433.

17. Min DI, Hwang GC, Bergstrom S et al. Bioavailability and patient acceptance of cyclosporine soft gelatin capsules in renal allograft recipients. Ann Pharmacother 1992; 26: 175.

18. Zehnder C, Beveridge T, Nuesch E, Abisch A, Thiel G. Cyclosporine A capsules: bioavailability and clinical acceptance study in renal transplant patients. Transplant Proc 1988; 20 (2 Suppl. 2): 641.

19. Bleck JS, Nashan B, Christians U, Schottmann R, Wonigeit K, Sewing KF. Single dose pharmacokinetics of ciclosporin and its main metabolites after oral ciclosporin as oily solution or capsule. Arzneimittelforschung 1990; 40: 62.

20. Nashan B, Bleck J, Wonigeit K et al. Effect of the application form of cyclosporine on blood levels: comparison of oral solution and capsules. Transplant Proc 1988; 20(2 Suppl. 2): 637.

21. Hilbrands LB, Hoitsma AJ, van den Berg JW, Koene RA. Cyclosporin $A$ blood levels during use of cyclosporin as oral solu- tion or in capsules: comparison of pharmacokinetic parameters. Transplant Int 1991; 4: 125

22. Bennett WM, Olyaei AJ. Pharmacoeconomics of immunosuppressive agents in renal transplant recipients. Transplant Proc 1999; 31(3A (Suppl.): 6S.

23. Coukell AJ, Plosker GL. Cyclosporin microemulsion (Neoral). A pharmacoeconomic review of its use compared with standard cyclosporin in renal and hepatic transplantation. Pharmacoeconomics 1998; 14: 691.

24. Alloway RR. Generic immunosuppressant use in solid organ transplantation. Transplant Proc 1999; 31(3A (Suppl.): 2S

25. Roza A, Tomlanovich S, Merion R et al. Conversion of stable renal allograft recipients to a bioequivalent cyclosporine formulation. Transplantation 2002; 74: 1013

26. Tomlanovich S, Pollack R, Roza A et al. A pharmacokinetic conversion study of Abbott cyclosporine and NEORAL in stable renal transplant recipients. North Chicago, IL: Abbott Laboratories, Inc. 1999; 60064: 99J-031-99J-8028.

27. Lindholm A, Welsh M, Rutzky L, Kahan BD. The adverse impact of high cyclosporine. Clearance rates on the incidences of acute rejection and graft loss. Transplantation 1993; 55: 985.

28. Merion RM, White DJ, Thiru S, Evans DB, Calne RY. Cyclosporine: five years' experience in cadaveric renal transplantation. N Engl $J$ Med 1984; 310: 148.

29. Neylan JF. Immunosuppressive therapy in high-risk transplant patients: dose-dependent efficacy of mycophenolate mofetil in African-American renal allograft recipients. U.S. Renal Transplant Mycophenolate Mofetil Study Group. Transplantation 1997; 64: 1277.

30. Watkins PB. The barrier function of CYP3A4 and P-glycoprotein in the small bowel. Adv Drug Deliv Rev 1997; 27: 161.

31. Malhotra S, Bailey DG, Paine MF, Watkins PB. Seville orange juice-felodipine interaction: comparison with dilute grapefruit juice and involvement of furocoumarins. Clin Pharmacol Ther 2001; 69: 14

32. Watkins $P$, Leichtman A. The molecular basis of cyclosporin a metabolism, pharmacokinetics and drug interactions. Graft 1999; 2: 177 .

33. Kasiske BL, Cohen D, Lucey MR, Neylan JF. Payment for immunosuppression after organ transplantation: American Society of Transplantation. JAMA 2000; 283: 2445.

34. Burckart G, Venkataramanan R, Starzl T, Ptachcinski R, Gartner C, Rosenthal $\mathrm{T}$. Cyclsoporine clearance in children following organ transplantation. J Clin Pharmacol 1985; 24: 412

35. Venkataramanan R, Burckhart GJ, Ptachcinski RJ. Pharmacokinetics and monitoring of cyclosporine following orthotopic liver transplantation. Semin Liver Dis 1985; 5: 357

36. Yee GC, Lennon TP, Gmur DJ, Kennedy MS, Deeg HJ. Agedependent cyclosporine: pharmacokinetics in marrow transplant recipients. Clin Pharmacol Ther 1986; 40: 438.

37. Hoyer PF, Brodehl J, Ehrich JH, Offner G. Practical aspects in the use of cyclosporin in paediatric nephrology. Pediatr Nephrol 1991; 5: 630

38. Isaacs RB. Optimal transplant immunosuppression: a case of the haves and have nots? Am J Kidney Dis 2001; 37: 160 